\title{
AXIOMS FOR SEQUENTIAL CONVERGENCE
}

\author{
GONÇALO GUTIERRES AND DIRK HOFMANN
}

\begin{abstract}
It is of general knowledge that those (ultra)filter convergence relations coming from a topology can be characterized by two natural axioms. However, the situation changes considerable when moving to sequential spaces. In case of unique limit points J. Kisyński [Kis60] obtained a result for sequential convergence similar to the one for ultrafilters, but the general case seems more difficult to deal with. Finally, the problem was solved by V. Koutnik [Kou85].

In this paper we present an alternative approach to this problem. Our goal is to find a characterization more related to the case of ultrafilter convergence. We extend then the result to characterize sequential convergence relations corresponding to Fréchet topologies, as well to those corresponding to pretopological spaces.
\end{abstract}

KEYWORDS: Convergence, sequence, sequential space, monad.

AMS Subject Classification (2000): 54D55, 54A20, 54B30, 18C15.

\section{Introduction}

The development of topology was from the beginning linked to the search of a good notion of convergence. Traditionally, convergence means sequential convergence, but it became soon clear that sequences are not adequate for the purpose of general topology. Therefore the further development of topology was based on other concepts like open set, closure operator or neighborhood system. Eventually, the concept of convergence was reintroduced into topology: using generalized sequences (nets) by Moore and Smith [SM22] and, later, (ultra)filters by Cartan [Car37b, Car37a]. This rises the question how to characterize abstractly those (ultra)filter (resp. net) convergence relations coming from a topology. The answer is known since the fifties (see [Kow54], for instance). Subsequently, the formulation of such axioms on an ultrafilter convergence relation was considerable streamlined, eventually one obtains a system of two simple axioms (see [Bar70, Wyl96]). In fact, Barr [Bar70] describes topological spaces as relational algebras for the ultrafilter monad. The theory of relational algebras gained recently renewed interest

Received September 1, 2005.

The authors acknowledge partial financial assistance by Centro de Matemática da Universidade de Coimbra/FCT and Unidade de Investigação e Desenvolvimento Matemática e Aplicações da Universidade de Aveiro/FCT. 
(see [CT03, CHT04, Sea05]), and was used to study uniformly various important categories of topology (see [CH04, CH05, Hof05b], for instance).

Though sequences do not suffice for topological spaces in general, the class of spaces where sequences do suffice still includes many important spaces, and therefore enjoys attention of many authors (see [Dud64, Bro74]). Naturally, we have the same question as above: How can we characterize those sequential convergence relations coming from a topology? An answer to this question seems to be much harder to find then in the case of ultrafilters. Eventually, the problem was solved by V. Koutnik [Kou85], but his new axiom $(\mathcal{B})$ is far away from the simplicity of the "ultrafilter axioms". Moreover, it is desirable to have a description closer to Barr's relational algebras, since it allows us to apply techniques developed in this setting to sequential spaces. To fulfil the latter requirement, we must first of all know what the sequential monad is.

The relationship between various basic concepts of topology can be most elegantly expressed via Galois correspondences, which is the starting point for this work. Our principal interest is then the study of the correspondence between open sets on one side and sequential convergence relations on the other. The fixed objects of this correspondence are sequential topologies* on the "open set side", whereby finding a description of the fixed elements on the convergence side answers exactly the question above. In case of unique convergence points, J. Kisyński [Kis60] gave a nice answer in form of three simple axioms (1), (2) and (3) (see 2.1), but this does not extend to the general case. Guided by our experience from the work with ultrafilters, in Section 2 we introduce diagonal sequences of sequences of sequences, which equips the "sequence functor" with the structure of a weak form of a monad. This allows us to introduce co-Kleisli composition for convergence relations - as it was done in [HT04] for the ultrafilter monad - and to formulate a new axiom (4) which corresponds to the second axiom (idempotency) in the topological case (see 2.3). But even $(1), \ldots,(4)$ do not suffice to describe sequential convergence, as we show in 2.4. This counter example indicates that $(1), \ldots,(4)$ are not strong enough to deal with certain sequences of sequences (and sequences of sequences of sequences, and so on). We respond to this difficulty by lifting axiom (3) to "higher order" sequences. This indeed leads

${ }^{*}$ Recall that a topological space $X$ is a sequential space if a subset $A$ of $X$ is open whenever each sequence $\left(x_{n}\right)_{n \in \mathbb{N}}$ with $\left(x_{n}\right)_{n \in \mathbb{N}} \rightarrow x \in A$ eventually belongs to $A$. A sequential space is a Fréchet space if the sequential closure induced by the topology is idempotent. 
us to the desired characterization (Theorem 3.3). En passant we show that $(1), \ldots$, (4) together with the "transitivity axiom" (5) describe Fréchet spaces (Theorem 2.5).

\section{Motivation}

1.1. Galois correspondences. Let $T:$ Set $\rightarrow$ Set be a functor which preserves monomorphisms. In particular, $T i$ is a monomorphism for every inclusion map $i: A \hookrightarrow X$. Hence we may think of $T A$ as a subset of $T X$ and consequently write $\mathfrak{x} \in T A$ for an element $\mathfrak{x}$ of $T X$ in the image of $T i$. Let now $X$ be a set and $a: T X \longrightarrow X$ be a relation. Given $\mathfrak{x} \in T X$ and $x \in X$, we think of $\mathfrak{x} a x$ as $\mathfrak{x}$ converges to $x$, and consequently write $\mathfrak{x} \rightarrow x$. A subset $A \subseteq X$ of $X$ is called a-open if

$$
\forall \mathfrak{x} \in T X \forall x \in X(\mathfrak{x} \rightarrow x \& x \in A) \Rightarrow \mathfrak{x} \in T A .
$$

The empty set as well as $X$ are $a$-open, and so are arbitrary unions of $a$-open subsets. The fact that $T(A \cap B)=T A \cap T B$ for all subsets $A, B$ of $X$ with $A \cap B \neq \emptyset$ (see [Man02] for a hint) implies that also finite intersections of $a$-open subsets are $a$-open, hence the collection $\mathcal{T}(a)$ of all $a$-open subsets of $X$ is actually a topology on $X$. Conversely, for each subset $\tau \subseteq P X$ (being a topology or not) of the powerset $P X$ of $X$ we define a relation $\mathcal{C}(\tau): T X \mapsto X$ by putting

$$
\mathfrak{x} \rightarrow x \quad: \Longleftrightarrow \quad \forall A \subseteq X(A \in \tau \& x \in A) \Rightarrow \mathfrak{x} \in T A
$$

for each $\mathfrak{x} \in T X$ and $x \in X$. We have constructed a pair of functions

$$
\{\tau \subseteq P X\} \underset{\mathcal{T}}{\mathcal{T}}\{a: T X \mapsto X\}
$$

which are easily seen to be order-reversing and satisfy $\tau \subseteq \mathcal{T C}(\tau)$ and $a \subseteq$ $\mathcal{C T}(a)$. In general, a pair of order-reversing functions $\varphi: X \rightarrow Y$ and $\psi:$ $Y \rightarrow X$ between ordered $\operatorname{sets}^{\dagger} X$ and $Y$ satisfying the inequalities $\operatorname{id}_{X} \leq \psi \varphi$ and $\operatorname{id}_{Y} \leq \varphi \psi$ is called Galois correspondence. The maps $\varphi$ and $\psi$ can be restricted to order-reversing isomorphisms between the subsets

$$
\operatorname{Fix}_{\psi \varphi}=\{x \in X \mid x=\psi \varphi(x)\} \quad \text { and } \quad \operatorname{Fix}_{\varphi \psi}=\{y \in Y \mid y=\varphi \psi(y)\} .
$$

Note that $\operatorname{Fix}_{\psi \varphi}$ is equal to the image of $\psi$ and, likewise, $\operatorname{Fix}_{\varphi \psi}=\operatorname{im} \phi$. We saw already that each element of Fix $\mathcal{T C}_{\mathcal{C}}$ is a topology on $X$.

\footnotetext{
${ }^{\dagger}$ Sets equipped with a reflexive and transitive relation $\leq$. Note that we do not require antisymmetry.
} 
1.2. Examples. We obtain the simplest example of such a situation by choosing $T=\mathrm{Id}:$ Set $\rightarrow$ Set. Then $\mathcal{C}$ associates to each subset $\tau$ of $P X$ its specialization order defined by

$$
x \rightarrow y \quad: \Longleftrightarrow \quad \forall A \subseteq X(A \in \tau \& y \in A) \Rightarrow x \in A .
$$

Here $\mathcal{C}(\tau)$ is indeed an order relation on $X$ and each order relation on $X$ is obtained that way. Therefore $\operatorname{Fix}_{\mathcal{C} \mathcal{T}}$ is the set of all order relations on $X$. Turning to the topological side, the elements of Fix $\operatorname{TC}_{\mathcal{C}}$ are exactly those topologies on $X$ which are determined by their specialization order. It is well-known that these spaces are exactly the Alexandroff spaces. Recall that a topology is Alexandroff if it is closed under arbitrary intersections.

The ultrafilter functor $U$ : Set $\rightarrow$ Set assigns to each set $X$ the set $U X$ of all ultrafilters on $X$ and to each function $f: X \rightarrow Y$ the function $U f: U X \rightarrow$ $U Y$ which takes an ultrafilter $\mathfrak{x} \in U X$ to the (ultra)filter generated by its $f$ image $\{f[A] \mid A \in \mathfrak{x}\}$. It is easy to see that $U$ preserves monomorphisms. Of course, Fix $_{\mathcal{T C}}$ consists now of all topologies on $X$, while an elegant description of $\operatorname{Fix}_{\mathcal{C} \mathcal{T}}$ requires further notation.

Our principal interest is the study of the correspondence obtained from a sequential functor $S:$ Set $\rightarrow$ Set. There are various ways to define $S$. By technical reasons we choose $S X=X^{\mathbb{N}} / \sim$ the set of all sequences on $X$ modulo the equivalence relation

$$
\left(x_{n}\right)_{n \in \mathbb{N}} \sim\left(y_{n}\right)_{n \in \mathbb{N}} \quad: \Longleftrightarrow\left\{n \in \mathbb{N} \mid x_{n} \neq y_{n}\right\} \text { is finite }
$$

and $S f\left[\left(x_{n}\right)_{n \in \mathbb{N}}\right]=\left[\left(f\left(x_{n}\right)\right)_{n \in \mathbb{N}}\right]$. The relation $\mathcal{C}(\tau): S X \longrightarrow X$ for $\tau \subseteq P X$ is now the expected one: $\left[\left(x_{n}\right)_{n \in \mathbb{N}}\right] \rightarrow x$ if and only if $\left(x_{n}\right)_{n \in \mathbb{N}}$ belongs eventually to each $A \in \tau$ with $x \in A$. It is clear that this condition does not depend on the particular representant $\left(x_{n}\right)_{n \in \mathbb{N}}$. To simplify notation, in the sequel we will just write $\left(x_{n}\right)_{n \in \mathbb{N}}$, rather than $\left[\left(x_{n}\right)_{n \in \mathbb{N}}\right]$, with the understanding that we mean the equivalence class. From the considerations above we see that the elements of $\mathrm{Fix}_{\mathcal{T C}}$ are exactly the sequential topologies on $X$.

We write $\left(y_{n}\right)_{n \in \mathbb{N}} \preccurlyeq\left(x_{n}\right)_{n \in \mathbb{N}}$ to indicate that $\left(y_{n}\right)_{n \in \mathbb{N}}$ is a subsequence of $\left(x_{n}\right)_{n \in \mathbb{N}}$. A little bit of care is needed here since we are talking about equivalence classes of sequences. We say that $\left(y_{n}\right)_{n \in \mathbb{N}}$ is a subsequence of $\left(x_{n}\right)_{n \in \mathbb{N}}$ if it is so beginning from some order. In other words, $\left(y_{n}\right)_{n \in \mathbb{N}}$ is equivalent to a subsequence of $\left(x_{n}\right)_{n \in \mathbb{N}}$ in the usual sense.

1.3. The category of relations. The category Rel has sets as objects, and a morphism from $X$ to $Y$ is a relation $r: X \mapsto Y$. Composition is given by 
relational composition:

$$
x(s \cdot r) z \quad: \Longleftrightarrow \quad \exists y \in Y \text { xry \& yrz }
$$

for relations $r: X \mapsto Y$ and $s: Y \longrightarrow Z$ and elements $x \in X$ and $z \in Z$. Then the identity arrow $\operatorname{id}_{X}: X \longrightarrow X$ is the equality relation on $X$. In contrast to Set, there is a non-trivial order on $\operatorname{Rel}(X, Y)$ given by inclusion. Each map $f: X \rightarrow Y$ can be seen as the relation $x f y \Longleftrightarrow f(x)=y$, and relational composition of two functions coincides with ordinary composition. The transpose $r^{\circ}: Y \longrightarrow X$ of a relation $r: X \mapsto Y$ is defined by $y r^{\circ} x: \Longleftrightarrow$ $x r y$. Of course, the transpose $f^{\circ}$ of a Set-map $f: X \rightarrow Y$ is just its inverse image relation. We remark that each $f: X \rightarrow Y$ satisfies the inequalities $\operatorname{id}_{X} \leq f^{\circ} \cdot f$ and $f \cdot f^{\circ} \leq \operatorname{id}_{Y}$, i.e. $f$ is left adjoint to $f^{\circ}$. Returning to our examples, we have that a relation $a: X \longrightarrow X$ belongs to Fix $_{\mathcal{C} \mathcal{T}}$ for the identity functor (that is, $a$ is an order relation) if and only if it satisfies

$$
\operatorname{id}_{X} \leq a \quad \text { and } \quad a \cdot a \leq a .
$$

This result cannot be extended directly to functors different from the identity since in general we cannot compose relations $a: T X \longrightarrow X$ and $b: T X \longrightarrow X$. However, some adicional structure on $T$ allows to define a useful composition.

1.4. Monads and co-Kleisli composition. Recall that a monad $\mathbb{T}=$ $(T, e, m)$ on Set consists of a functor $T:$ Set $\rightarrow$ Set together with natural transformations $e:$ Id $\rightarrow T$ (unit) and $m: T T \rightarrow T$ (multiplication) such that the diagrams
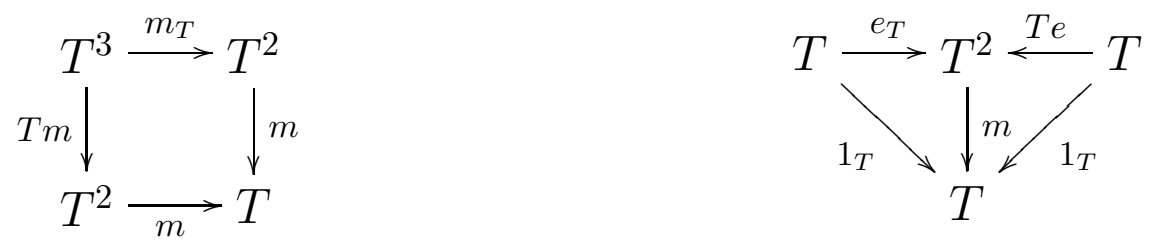

commute. The identity monad consisting of the identity functor and identity transformations is a trivial example of a monad. But also the ultrafilter functor $U$ can be made into a monad $\mathbb{U}=(U, e, m)$. The natural transformations $e: \operatorname{Id} \rightarrow U$ and $m: U^{2} \rightarrow U$ are given by

$$
e_{X}(x)=\dot{x}=\{A \subseteq X \mid x \in A\} \quad \text { and } \quad m_{X}(\mathfrak{X})=\left\{A \subseteq X \mid \mathfrak{X} \in U^{2} A\right\},
$$


for all sets $X, \mathfrak{X} \in U^{2} X$ and $x \in X$. Every relation $r: X \mapsto Y$ gives a relation $\operatorname{Tr}: T X \longrightarrow T Y$ defined by

$$
\mathfrak{x}(T r) \mathfrak{y} \quad: \Longleftrightarrow \quad \exists \mathfrak{w} \in T\left(G_{r}\right)\left(T \pi_{X}(\mathfrak{w})=\mathfrak{x} \& T \pi_{Y}(\mathfrak{w})=\mathfrak{y}\right),
$$

where $G_{r} \subseteq X \times Y$ is the graph of $r$. For later use we describe explicitly the extension $S:$ Rel $\rightarrow$ Rel of the sequential functor $S$. For a relation $r: X \longrightarrow Y$ and sequences $\left(x_{n}\right)_{n \in \mathbb{N}} \in S X$ and $\left(y_{n}\right)_{n \in \mathbb{N}} \in S Y$, we have $\left(x_{n}\right)_{n \in \mathbb{N}}(S r)\left(y_{n}\right)_{n \in \mathbb{N}}$ if and only if $x_{n} r y_{n}$ for almost all $n \in \mathbb{N}$. For an arbitrary functor $T:$ Set $\rightarrow$ Set we obtain only $T(s \cdot r) \leq T s \cdot T r$ for relations $r: X \longrightarrow$ ' $Y$ and $s: Y \longrightarrow Z$, however, in case $T=\operatorname{Id}, U$ or $S$ we have equality. The natural transformations $e$ and $m$ become op-lax, that is, for every relation $r: X \mapsto Y$ we have the inequalities

$$
\begin{array}{cr}
e_{Y} \cdot r \leq T r \cdot e_{X}, & m_{Y} \cdot T T r \leq T r \cdot m_{X} . \\
X \stackrel{e_{X}}{\longrightarrow} T X & T T X \stackrel{m_{X}}{\longrightarrow} T X \\
r \downarrow \leq \downarrow T r & T T r \downarrow \leq \downarrow T r \\
Y \underset{e_{Y}}{\longrightarrow} T Y & T T Y \underset{m_{Y}}{\longrightarrow} T Y
\end{array}
$$

In our examples $T=\mathrm{Id}$ and $T=U$ we have even $m_{Y} \cdot T T r=T r \cdot m_{X}$.

Let $a: T X \mapsto X$ and $b: T X \rightarrow X$ be relations. We define the co-Kleisli composition of $b$ and $a$ as

$$
b * a:=b \cdot T a \cdot m_{X}^{\circ} .
$$

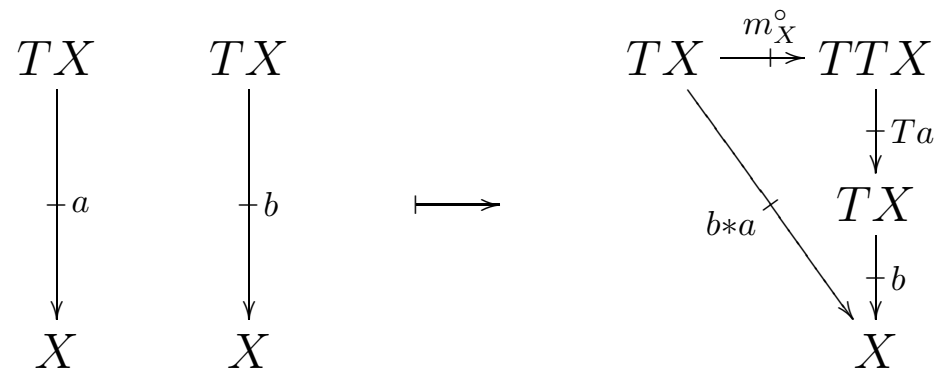

For each $a: T X \longrightarrow Y$ we have $a * e_{X}^{\circ}=a$ and $e_{Y}^{\circ} * a \geq a$, that is, the relation $e_{X}^{\circ}$ is a lax identity for this composition. We have developed now the language to describe the elements of $\operatorname{Fix}_{\mathcal{C} \mathcal{T}}$ for the ultrafilter monad $\mathbb{U}$ exactly in the same style as for the identity monad: $a: U X \mapsto X$ belongs to $\operatorname{Fix}_{\mathcal{C}}$ if and only if

$$
e_{X}^{\circ} \leq a \text { (Extensivity) and } \quad a * a \leq a \text { (Idempotency). }
$$


Note that the first inequality forces the second one to be an equality. This presentation of Fix $\mathcal{T C}_{\mathcal{C}}$ is described in detail in [HT04]. The adjointness inequalities for the functions $e_{X}$ and $m_{X}$ make the inequalities above equivalent to

$$
\operatorname{id}_{X} \leq a \cdot e_{X} \text { (Reflexivity)) and } a \cdot T a \leq a \cdot m_{X} \text { (Transitivity) }
$$

respectively, which let $a: U X \longrightarrow X$ look like a lax Eilenberg-Moore algebra structure for $\mathbb{T}$. These algebras are introduced in [Bar70] for an arbitrary monad $\mathbb{T}$ under the name relational algebras, and it is shown that for $\mathbb{T}=\mathbb{U}$ they describe exactly topological spaces. We are seeking for a similar result for $T=S$, however, so far we do not know what the monad structure for $S$ is. Of course, for each set $X$ there is a natural map $e_{X}: X \rightarrow S X, x \mapsto \dot{x}:=$ $[(x)]$, but there seems to be no canonical candidate for the multiplication $m$. It is the aim of the next section to find such a candidate.

\section{The sequential monad}

2.1. When convergence is unique. We start by recalling a result of Kisyński [Kis60] which characterizes the partial functions belonging to $\mathrm{Fix}_{\mathcal{C} \mathcal{T}}$. A convergence space $(X, a)$ is called $\mathcal{L}^{*}$-space if

(0) each $\left(x_{n}\right)_{n \in \mathbb{N}}$ has at most one convergence point,

(1) $\dot{x} \rightarrow x$,

(2) $\left(x_{n}\right)_{n \in \mathbb{N}} \rightarrow x \Longrightarrow\left(\forall\left(y_{n}\right)_{n \in \mathbb{N}} \preccurlyeq\left(x_{n}\right)_{n \in \mathbb{N}}\left(y_{n}\right)_{n \in \mathbb{N}} \rightarrow x\right)$,

(3) $\left(\forall\left(y_{n}\right)_{n \in \mathbb{N}} \preccurlyeq\left(x_{n}\right)_{n \in \mathbb{N}} \exists\left(z_{n}\right)_{n \in \mathbb{N}} \preccurlyeq\left(y_{n}\right)_{n \in \mathbb{N}}\left(z_{n}\right)_{n \in \mathbb{N}} \rightarrow x\right) \Longrightarrow$ $\left(x_{n}\right)_{n \in \mathbb{N}} \rightarrow x$

for all $x \in X$ and $\left(x_{n}\right)_{n \in \mathbb{N}} \in S X$.

Theorem ([Kis60]). Let $(X, a)$ be a convergence space satisfying (0). Then $(X, a)$ is a $\mathcal{L}^{*}$-space if and only if a is a fixed point of the Galois correspondence.

The corresponding topological spaces are the sequentially Hausdorff sequential spaces. It can be easily seen that $\mathcal{C}(\tau)$ satisfies the conditions (1), (2) and (3) for each $\tau \in P X$, hence each $a \in$ Fix $_{\mathcal{C}}$ must satisfy them. However, these conditions are in general not sufficient to guarantee $a \in \operatorname{Fix}_{\mathcal{C} \mathcal{T}}$ as the following example shows.

Example. Let $X=\{1,2,3\}$ and define

$$
\left(x_{n}\right)_{n \in \mathbb{N}} \rightarrow x \quad: \Longleftrightarrow \quad\left(x_{n}\right)_{n \in \mathbb{N}} \in S(\{x, x+1\} \cap X) .
$$


Then (1), (2) and (3) are fulfilled. We have $\dot{3} \rightarrow 2$ and $\dot{2} \rightarrow 1$ but not $\dot{3} \rightarrow 1$, which shows that this convergence on $X$ cannot come from a topology.

The insufficiency of (1), (2) and (3) does not come as a surprise since we do not require any diagonal condition yet.

2.2. Natural order on $T X$. We learned from [Sea05] that for each monomorphism preserving functor $T:$ Set $\rightarrow$ Set there is a canonical order relation on $T X$ :

$$
\mathfrak{x} \geq \mathfrak{y} \quad: \Longleftrightarrow \quad \forall A \subseteq X(\mathfrak{x} \in T A \Longrightarrow \mathfrak{y} \in T A)
$$

for all $\mathfrak{x}, \mathfrak{y} \in T X$. In the examples discussed so far we obtain the expected order: the equality relation if $T=\operatorname{Id}$ or $T=U$, whereby for $T=S$ we have $\left(x_{n}\right)_{n \in \mathbb{N}} \geq\left(y_{n}\right)_{n \in \mathbb{N}}$ if and only if, for each $N \in \mathbb{N}$, there exists some $M \in \mathbb{N}$ such that $\left\{y_{n} \mid n \geq M\right\} \subseteq\left\{x_{n} \mid n \geq N\right\}$.

This construction can be generalized further. Given monomorphism preserving functors $R, T$ : Set $\rightarrow$ Set, as above we define, for each set $X$, a relation $k_{X}: R X \longrightarrow T X$ by putting

$$
\mathfrak{x}\left(k_{X}\right) \mathfrak{y} \quad: \Longleftrightarrow \forall A \subseteq X \quad(\mathfrak{x} \in R A \Longrightarrow \mathfrak{y} \in T A)
$$

for all $\mathfrak{x} \in R X$ and $\mathfrak{y} \in T X$. For instance, if we choose $T=U$ and $R=\operatorname{Id}$ or $R=U^{2}$, we obtain exactly the unit and the multiplication of the ultrafilter monad. This encourages us to define diagonal sequences in the same way.

2.3. Diagonal sequences. Motivated by the considerations above, we say that $\mathfrak{x}=\left(x_{n}\right)_{n \in \mathbb{N}} \in S X$ is a diagonal sequence of $\mathfrak{X}=\left(x_{m}^{n}\right)_{n, m \in \mathbb{N}} \in S S X$ (written as $\left.\mathfrak{X}\left(m_{X}\right) \mathfrak{x}\right)$ if, for all $A \subseteq X, \mathfrak{X} \in S S A$ implies $\mathfrak{x} \in S A$. Hence $\left(x_{n}\right)_{n \in \mathbb{N}}$ is a diagonal of $\left(x_{m}^{n}\right)_{n, m \in \mathbb{N}}$ if and only if, for each $N \in \mathbb{N}$ and each family $\left(m_{n}\right)_{n \geq N}$ of natural numbers, there exists $M \in \mathbb{N}$ such that $\left\{x_{n}\right.$ | $n \geq M\} \subseteq\left\{x_{n, m} \mid n \geq N, m \geq m_{n}\right\}$. At this point it becomes clear why we consider equivalence classes of sequences and not just the functor $S^{\prime} X=X^{\mathbb{N}}$. In the latter case we obtain that $\left(x_{n}\right)_{n \in \mathbb{N}}$ is a diagonal of $\left(x_{m}^{n}\right)_{n, m \in \mathbb{N}}$ if and only if $\left\{x_{n} \mid n \in \mathbb{N}\right\} \subseteq\left\{x_{n, m} \mid n, m \in \mathbb{N}\right\}$. This diagonal considers only the set of elements of a sequence regardless of their position in the sequence, hence it is not the "right one" for our study of sequential convergence in general. However, it turns out to be useful in the case of Fréchet spaces (see $2.5)$. 
Note that $m_{X}: S S X \longrightarrow S X$ is in general just a relation and not a function as in the examples above. In fact, it is a very weak relation in the sense that most of the double sequences do not have any diagonal sequence at all. However, we have that
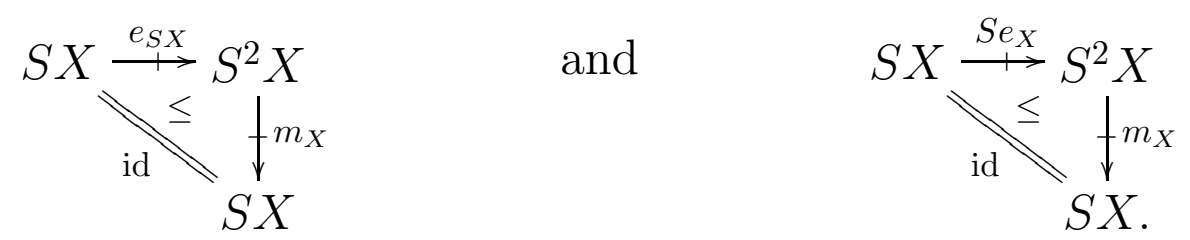

In other words, every sequence $\mathfrak{x}=\left(x_{n}\right)_{n \in \mathbb{N}}$ is diagonal to the double sequence $e_{S X}(\mathfrak{x})=\left(\dot{x_{n}}\right)_{n \in \mathbb{N}}$ which is constant $\left(x_{n}\right)_{n \in \mathbb{N}}$ as well as to the double sequence $S e_{X}(\mathfrak{x})=\left(\dot{x}_{n}\right)_{n \in \mathbb{N}}$ formed by the constant sequences $\dot{x}_{n}$. We add now the idempotency axiom $a * a \leq a$ to our list. In this setting it reads as

(4) $\left[\left(x_{m}^{n}\right)_{m, n \in \mathbb{N}} \stackrel{m}{\longrightarrow}\left(y_{n}\right)_{n \in \mathbb{N}} \stackrel{n}{\longrightarrow} x\right]$ and $\left[\left(x_{m}^{n}\right)_{m, n \in \mathbb{N}}\left(m_{X}\right)\left(x_{k}\right)_{k \in \mathbb{N}}\right] \Longrightarrow$ $\left(x_{k}\right)_{k \in \mathbb{N}} \rightarrow x$.

We remark that $a * a \leq a$ is not equivalent to $a \cdot S a \leq a \cdot m_{X}$, since $m_{X}$ is in general not a function. It is easy to see that (1) and (4) together imply (2). We show now that (4) can be reduced to two simpler axioms. First we state some auxiliary results.

Lemma. Let $X$ be a set and $a: S X \rightarrow X$. Then the following assertions hold.

(a) Assume that $(X, a)$ satisfies (2) and (3). Let $\left(x_{n}\right)_{n \in \mathbb{N}} \rightarrow x$ in $(X, a)$ and $\varphi: \mathbb{N} \rightarrow \mathbb{N}$ be a bijection. Then $\left(x_{\varphi(n)}\right)_{n \in \mathbb{N}} \rightarrow x$.

(b) Let $\left(x_{m}^{n}\right)_{n, m \in \mathbb{N}} \in S^{2} X$ and $\left(\varphi_{n}: \mathbb{N} \rightarrow \mathbb{N}\right)_{n \in \mathbb{N}}$ be a family of bijections. If $\left(x_{n}\right)_{n \in \mathbb{N}}$ is a diagonal of $\left(x_{m}^{n}\right)_{n, m \in \mathbb{N}}$, then it is a diagonal of $\left(x_{\varphi(m)}^{n}\right)_{n, m \in \mathbb{N}}$ as well.

Proposition. Under (1), (2), (3), axiom (4) is equivalent to (4a) $\left(\dot{x}_{n}\right)_{n \in \mathbb{N}} \rightarrow\left(y_{n}\right)_{n \in \mathbb{N}} \rightarrow x \Rightarrow\left(x_{n}\right)_{n \in \mathbb{N}} \rightarrow x \quad$ (that is: $\left.a \cdot S a \cdot S e_{X} \leq a\right)$, (4b) $\left(\dot{x_{m}}\right)_{m \in \mathbb{N}} \rightarrow\left(y_{n}\right)_{n \in \mathbb{N}} \rightarrow x \Rightarrow\left(x_{n}\right)_{n \in \mathbb{N}} \rightarrow x \quad$ (that is: $\left.a \cdot S a \cdot e_{S X} \leq a\right)$. Proof: Clearly, (4) implies (4a) and (4b) since $\left(x_{n}\right)_{n \in \mathbb{N}}$ is a diagonal sequence of both $\left(\dot{x}_{n}\right)_{n \in \mathbb{N}}$ and $\left(\dot{x}_{n}\right)_{n \in \mathbb{N}}$. Suppose now that $\left(x_{m}^{n}\right)_{m, n \in \mathbb{N}} \rightarrow\left(y_{n}\right)_{n} \rightarrow x$ and $\left(x_{n}\right)_{n \in \mathbb{N}}$ is a diagonal sequence of $\left(x_{m}^{n}\right)_{m, n \in \mathbb{N}}$. From (2) it follows that we can assume that $\left\{x_{n} \mid x \in \mathbb{N}\right\}=\left\{x_{m}^{n} \mid n, m \in \mathbb{N}\right\}$. According to 
(3), we must show that each subsequence of $\left(x_{n}\right)_{n \in \mathbb{N}}$ contains a subsequence which converges to $x$. Since each subsequence of $\left(x_{n}\right)_{n \in \mathbb{N}}$ is a diagonal of $\left(x_{m}^{n}\right)_{m, n \in \mathbb{N}}$ as well, it is enough to show that $\left(x_{n}\right)_{n \in \mathbb{N}}$ contains a subsequence which converges to $x$. Furthermore, since each sequence contains either a constant or an injective subsequence, we assume that $\left(x_{n}\right)_{n \in \mathbb{N}}$ is injective or constant. In the latter case we obtain immediately $\left(x_{n}\right)_{n \in \mathbb{N}} \rightarrow x$ from any of the conditions (4a) and (4b), since $\left(x_{m}^{n}\right)_{m, n \in \mathbb{N}}$ is constant too.

Assume now that $\left(x_{n}\right)_{n \in \mathbb{N}}$ is an injective sequence. As above, each of the sequences $\left(x_{m}^{n}\right)_{m \in \mathbb{N}}(n \in \mathbb{N})$ either has a constant subsequence or an injective one. Suppose first that $\mathbb{P}=\left\{n \in \mathbb{N} \mid\left(x_{m}^{n}\right)_{m \in \mathbb{N}}\right.$ has a constant subsequence $\}$ is infinite. Then, by (4a), every sequence $\left(z_{n}\right)_{n \in \mathbb{P}}$ converges to $x$, where $z_{n}$ appears infinitely many times in $\left(x_{m}^{n}\right)_{m \in \mathbb{N}}$. If we are able to choose $\left(z_{n}\right)_{n \in \mathbb{P}}$ so that $\left\{z_{n} \mid n \in \mathbb{P}\right\}$ is infinite, we conclude the proof. If not, then, beginning from some order $p \in \mathbb{P}$, there is only a finite set $\left\{z_{1}, \ldots, z_{k}\right\}$ of possible constants. We delete the sequences $\left(x_{m}^{1}\right)_{m \in \mathbb{N}}, \ldots,\left(x_{m}^{p-1}\right)_{m \in \mathbb{N}}$ and all elements in $\left\{z_{1}, \ldots, z_{k}\right\}$ from $\left(x_{m}^{n}\right)_{m, n \in \mathbb{N}}$. Then $\left(x_{n}\right)_{n \in \mathbb{N}}$ is still a diagonal of the so obtained sequence of sequences, and the next case applies. Assume now that $\left(x_{m}^{n}\right)_{m \in \mathbb{N}}$ is essentially injective, for almost all $n \in \mathbb{N}$. We can safely assume that $\left(x_{m}^{n}\right)_{m \in \mathbb{N}}$ is injective and, by the lemma above, we may even assume that $\left(x_{m}^{n}\right)_{m \in \mathbb{N}} \preccurlyeq\left(x_{k}\right)_{k \in \mathbb{N}}$, for all $n \in \mathbb{N}$. Since $\left(x_{m}^{1}\right)_{m \in \mathbb{N}}$ is a subsequence of $\left(x_{k}\right)_{k \in \mathbb{N}}$, it is also a diagonal of $\left(x_{m}^{n}\right)_{m, n}$. This fact implies that there is $n_{2}>1$ such that $\left(x_{m}^{n_{2}}\right)_{m \in \mathbb{N}}$ and $\left(x_{m}^{1}\right)_{m \in \mathbb{N}}$ have a common subsequence $\mathfrak{x}_{2}$. Iterating this process, we obtain a sequence of sequences $\left(\mathfrak{x}_{l}\right)_{l \in \mathbb{N}}$ in $X$ where $\mathfrak{x}_{l+1} \preccurlyeq \mathfrak{x}_{l}$ and $\mathfrak{x}_{l} \preccurlyeq\left(x_{m}^{n_{l}}\right)_{m \in \mathbb{N}}$. We define now $z_{1}=x_{m}^{1}$ and $z_{n+1}$ is the first element of $\mathfrak{x}_{n+1}$ different from $z_{1}, \ldots, z_{n}$. By construction, for all $l \in \mathbb{N}$, $\left(z_{n}\right)_{n \in \mathbb{N}} \preccurlyeq \mathfrak{x}_{l} \preccurlyeq\left(x_{m}^{n_{l}}\right)_{m \in \mathbb{N}} \preccurlyeq\left(x_{k}\right)_{k \in \mathbb{N}}$. Applying (4b), we obtain $\left(z_{n}\right)_{n \in \mathbb{N}} \rightarrow x$ which concludes the argument.

It is easy to see that each $\mathcal{C}(\tau)$ satisfies also (4). However, $(1), \ldots,(4)$ are still not sufficient to characterize $\operatorname{Fix}_{\mathcal{C} \mathcal{T}}$, as we show in the next subsection.

2.4. A counter example. We construct now a convergence space $(X, a)$ which satisfies $(1), \ldots,(4)$ and where $a$ is not induced by a topology on $X$. This counter example motivates the modification of axiom (3) studied in the next section.

Let $X=\mathbb{N} \cup P_{\text {inf }} \mathbb{N} \cup\{\star\}$, where $P_{\text {inf }} \mathbb{N}$ is the set of infinite subsets of $\mathbb{N}$. For $A, B \subseteq \mathbb{N}$, we define $A \leq B$ if $A \backslash B$ is finite. The set $X$ is equipped with the following convergence structure. 
(a) $\dot{x} \rightarrow x$, for all $x \in X$.

From now we consider only sequences with no constant subsequences.

(b) Let $\left(x_{n}\right)_{n \in \mathbb{N}} \in S \mathbb{N}$. Then $\left(x_{n}\right)_{n \in \mathbb{N}} \rightarrow A$ if $\left\{x_{n} \mid n \in \mathbb{N}\right\} \leq A$.

(c) Let $\left(A_{n}\right)_{n \in \mathbb{N}} \in S\left(P_{\text {inf }} \mathbb{N}\right)$. Then $\left(A_{n}\right)_{n \in \mathbb{N}} \rightarrow \star$ if

$$
\forall B \in P_{\text {inf }} \mathbb{N} \exists k \in \mathbb{N} \forall n \geq k \quad B \not A_{n} .
$$

There are no other convergences. One easily verifies (1), (2), (3), (4a) and (4b).

By definition, no sequence in $\mathbb{N}$ converges to $\star$. On the other hand, let $A$ be an infinite subset of $\mathbb{N}$. From $A \cong \mathbb{N} \times \mathbb{N}$ we obtain a sequence of sequences in $A$ converging to a sequence in $P_{\text {inf }} \mathbb{N}$, which in turn converges to $\star$. This shows that $\star$ belongs to the closure of each infinite subset of $\mathbb{N}$, hence $(n)_{n \in \mathbb{N}}$ converges to $\star$ in the induced topology.

Why do we have this counter example? The main reason is that we have a sequence of limit points of subsequences of $(n)_{n \in \mathbb{N}}$ converging to a point to which $(n)_{n \in \mathbb{N}}$ does not converge. To avoid this problem, it is useful to have a second order version of axiom (3):

$$
\text { (3.2) }\left[\forall\left(y_{k}\right)_{k \in \mathbb{N}} \preccurlyeq\left(x_{n}\right)_{n \in \mathbb{N}} \exists\left(z_{m}^{n}\right)_{m, n \in \mathbb{N}} \preccurlyeq\left(y_{k}\right)_{n \in \mathbb{N}}\left(z_{m}^{n}\right)_{n \in \mathbb{N}} \rightarrow x\right] \underset{\left(x_{n}\right)_{n \in \mathbb{N}}}{\Longrightarrow} \rightarrow x .
$$

By $\left(z_{m}^{n}\right)_{m, n \in \mathbb{N}} \preccurlyeq\left(y_{k}\right)_{k \in \mathbb{N}}$ we mean that every sequence $\left(z_{m}^{n}\right)_{m \in \mathbb{N}}$ is a subsequence of $\left(y_{k}\right)_{k \in \mathbb{N}}$, for almost all $n \in \mathbb{N}$ (see also Subsection 3.2). Clearly, (3.2) and (1) imply (3). However, it is possible to construct a convergence structure where (1), (2), (3.2) and (4) are satisfied, but it is still not a convergence structure of a topological space. This indicates that it is necessary to consider a more general version of axiom (3). We will do so in Section 3. Finally, for later use we point out that (3.2) implies (4b).

2.5. Fréchet spaces. Although adding axiom (4) to the previous three is not sufficient to characterize sequential spaces, it is very helpful to characterize convergence relations of Fréchet spaces. As before, the partial functions belonging to $\operatorname{Fix}_{\mathcal{C} T}$ and inducing a Fréchet topology are well known [Eng89, p. 64]. These spaces are called $\mathcal{S}^{*}$-spaces, and are the $\mathcal{L}^{*}$-spaces satisfying in addition

$$
\begin{aligned}
{\left[\left(x_{m}^{n}\right)_{n, m \in \mathbb{N}} \stackrel{m}{\longrightarrow}\left(y_{n}\right)_{n \in \mathbb{N}} \stackrel{n}{\longrightarrow} x\right] \Longrightarrow } \\
\forall A \subseteq X\left(\left(x_{m}^{n}\right)_{n, m \in \mathbb{N}} \in S^{2} A \Longrightarrow \exists\left(x_{k}\right)_{k \in \mathbb{N}} \in S A\left(x_{k}\right)_{k \in \mathbb{N}} \rightarrow x\right) .
\end{aligned}
$$


Axiom (5) essentially states that the sequential closure induced by the convergence space is idempotent.

Remark. The formulation of axiom (5) suffers some complications due to the fact that we consider equivalence classes of sequences. Of course, $A \subseteq X$ with $\left(x_{m}^{n}\right)_{n, m \in \mathbb{N}} \in S^{2} A$ is essentially $\left\{x_{n, m} \mid n, m \in \mathbb{N}\right\}$. Moreover, when considering $S^{\prime}$ and the corresponding diagonal relation $m_{X}^{\prime}$ (see 2.3), then $\left(x_{k}\right)_{k \in \mathbb{N}}$ is just a diagonal of $\left(x_{m}^{n}\right)_{n, m \in \mathbb{N}}$. We find it interesting to observe that, in this notation, (5) can be written as the transitivity condition $a \cdot S^{\prime} a \leq$ $a \cdot m_{X}^{\prime}$.

We have seen before that (1),..,(4) do not characterize sequential spaces, basically since we cannot deal with certain sequences of subsequences. However, the problem disappears if we add (5), since then (3.2) is provable. Having said this, our next theorem does not come as a surprise.

Theorem. A convergence space is a convergence of a Fréchet space if and only if satisfies (1), (2), (3), (4) and (5).

The proof of this theorem is not hard, moreover, it is a consequence of Theorem 3.3. For these reasons we do not include a proof here. We notice that (4) can be replaced by (4a), because (3) and (5) imply (3.2), which in turn implies (4b).

\section{The characterization}

3.1. Iteration of monads. The insufficiency of $(1), \ldots,(4)$ has its roots in the fact that the sequential closure is not idempotent. To make it idempotent requires one to consider sequences of sequences, and so on. This fact must be reflected in our axioms.

We define, for all ordinals $\alpha \leq \beta$, functors $S^{\alpha}$ : Set $\rightarrow$ Set and natural transformations $e^{\alpha, \beta}: S^{\alpha} \rightarrow S^{\beta}$ by putting

$$
\begin{array}{ll}
S^{0}=\mathrm{Id}, & \\
S^{\alpha+1}=S S^{\alpha}, & e^{\alpha, \alpha+1}=e_{S^{\alpha}}, \\
S^{\lambda}=\operatorname{colim}_{\alpha<\lambda} S^{\alpha}, & e^{\alpha, \lambda}=\text { colimit injection. }
\end{array}
$$

The latter colimit is taken pointwise in Set. It is important to observe that it is also a lax colimit in Rel in the following sense. For every family $\left(c_{\alpha}\right.$ : $\left.S^{\alpha} X \mapsto Z\right)_{\alpha<\lambda}$ satisfying $c_{\alpha+1} \cdot e_{X}^{\alpha, \alpha+1} \geq c_{\alpha}$, there is a relation $c: S^{\lambda} X \mapsto Z$ such that $c \geq c_{\alpha}$ for each ordinal $\alpha<\lambda$. Moreover, $c$ is universal with this 
property: it holds $c \leq c^{\prime}$ for any $c^{\prime}: S^{\lambda} X \mapsto Z$ such that $c^{\prime} \geq c_{\alpha}$ for each ordinal $\alpha<\lambda$. We denote the induced relation $c$ by $\left[c_{\alpha}\right]_{\alpha<\lambda}$. Recall that Rel is selfdual, hence $\left(\left(e_{X}^{\alpha, \lambda}\right)^{\circ}: S^{\lambda} X \longrightarrow S^{\alpha} X\right)_{\alpha<\lambda}$ is a lax limit in Rel. The induced relation $d: Z \longrightarrow S^{\lambda} X$ of a family $\left(d_{\alpha}: Z \longrightarrow S^{\alpha} X\right)_{\alpha<\lambda}$ is denoted by $\left\langle d_{\alpha}\right\rangle_{\alpha<\lambda}$.

Given a reflexive $a: S X \mapsto X$, we define relations $a^{\alpha}: S^{\alpha} X \mapsto X$ by putting

$$
a^{0}=a \cdot e_{X}, \quad a^{\alpha+1}=a \cdot S a^{\alpha}, \quad a^{\lambda}=\left[a^{\alpha}\right]_{\alpha<\lambda .}
$$

In the limit step we make use of the fact that $\left(a^{\alpha}\right)_{\alpha<\lambda}$ forms a lax cocone in the sense above. We extend the notation used so far and write $\mathfrak{x} \rightarrow x$ instead of $\mathfrak{x}\left(a^{\alpha}\right) x$, for $\mathfrak{x} \in S^{\alpha} X$ and $x \in X$. For $A \subseteq X$, we define $\sigma(A)=\{x \in X \mid$ $\left.\exists\left(x_{n}\right)_{n \in \mathbb{N}} \in S A\left(x_{n}\right)_{n \in \mathbb{N}} \rightarrow x\right\}$ and put

$$
\sigma^{\alpha+1}(A)=\sigma \cdot \sigma^{\alpha}(A), \quad \sigma^{\lambda}(A)=\bigcup_{\alpha<\lambda} \sigma^{\alpha}(A), \quad \hat{\sigma}(A)=\sigma^{\omega_{1}}(A) .
$$

Lemma. Let $a: S X \mapsto X$ be reflexive and $A \subseteq X$. For every ordinal $\alpha$ and $x \in X$, the following are equivalent:

(a) $x \in \sigma^{\alpha}(A)$;

(b) $\exists \mathfrak{x} \in S^{\alpha} A \mathfrak{x} \rightarrow x$.

Suppose that $(X, a)$ satisfies (1). It is not hard to see that the closure of $A \subseteq X$ in the induced topology is given by $\hat{\sigma}(A)$. Moreover, a sequence $\left(x_{n}\right)_{n \in \mathbb{N}}$ converges to $x$ in the induced topology if and only if $x$ belongs to the closure of $\left\{x_{n_{k}} \mid k \in \mathbb{N}\right\}$, for each subsequence $\left(x_{n_{k}}\right)_{k \in \mathbb{N}}$ of $\left(x_{n}\right)_{n \in \mathbb{N}}$. Hence we have the following

Proposition. For $a: S X \mapsto X$, the following are equivalent:

(a) $a \in \operatorname{Fix}_{\mathcal{C T}}$;

(b) a satisfies (1) and

$$
\left(\forall \mathfrak{y} \preccurlyeq\left(x_{n}\right)_{n \in \mathbb{N}} \forall A \subseteq X\left(\mathfrak{y} \in S A \Rightarrow \exists \alpha \exists \mathfrak{z} \in S^{\alpha} A \mathfrak{z} \rightarrow x\right)\right) \Rightarrow\left(x_{n}\right)_{n \in \mathbb{N}} \rightarrow x .
$$

We will show that $(*)$ can be substituted by (2), (4a) and a "higher order" version of (3). 
3.2. Higher order sequences. First we extend the subsequence relation $\preccurlyeq$ to "higher order sequences".

(a) For $x, y \in X$ we put $x \preccurlyeq y$ if $x=y$.

(b) For $\left(\mathfrak{x}_{n}\right)_{n \in \mathbb{N}},\left(\mathfrak{y}_{n}\right)_{n \in \mathbb{N}} \in S^{\alpha+1} X$ we define $\left(\mathfrak{x}_{n}\right)_{n \in \mathbb{N}} \preccurlyeq\left(\mathfrak{y}_{n}\right)_{n \in \mathbb{N}}$ if there exists some subsequence $\left(\mathfrak{y}_{n_{k}}\right)_{k \in \mathbb{N}}$ of $\left(\mathfrak{y}_{n}\right)_{n \in \mathbb{N}}$ such that, for each $k \in \mathbb{N}$, $\mathfrak{x}_{k} \preccurlyeq \mathfrak{y}_{n_{k}}$.

(c) $\preccurlyeq: S^{\lambda} X \mapsto S^{\lambda} X$ is the lax colimit of $\left(\preccurlyeq: S^{\alpha} X \mapsto S^{\alpha} X\right)_{\alpha<\lambda}$.

We are now able to introduce a "higher order" version of (3):

$\left(3^{\prime}\right)\left(\forall \mathfrak{y} \preccurlyeq\left(x_{n}\right)_{n \in \mathbb{N}} \exists \alpha \exists \mathfrak{z} \preccurlyeq e_{X}^{1, \alpha}(\mathfrak{y}) \mathfrak{z} \rightarrow x\right) \Rightarrow\left(x_{n}\right)_{n \in \mathbb{N}} \rightarrow x$.

One observes immediately that our new axiom $\left(3^{\prime}\right)$ is very similar to $\left(^{*}\right)$. The main difference is that here $\mathfrak{z}$ has to respect the order of the elements in $\mathfrak{y}$ instead of being merely formed by elements of $\mathfrak{y}$. We will show that (3') together with (1), (2) and (4a) indeed characterize Fix $x_{\mathcal{C}}$. To do so, we need some further notation.

We define relations $\#: S^{\alpha} X \mapsto S^{\alpha} X$ as follows.

(a) For $x, y \in X$ we put $x \# y$ if $x=y$.

(b) For $\left(\mathfrak{x}_{n}\right)_{n \in \mathbb{N}},\left(\mathfrak{y}_{m}\right)_{m \in \mathbb{N}} \in S^{\alpha+1} X$ we define $\left(\mathfrak{x}_{n}\right)_{n \in \mathbb{N}} \#\left(\mathfrak{y}_{m}\right)_{m \in \mathbb{N}}$ if there exist subsequences $\left(\mathfrak{x}_{n_{k}}\right)_{k \in \mathbb{N}}$ and $\left(\mathfrak{y}_{m_{k}}\right)_{k \in \mathbb{N}}$ of $\left(\mathfrak{x}_{n}\right)_{n \in \mathbb{N}}$ and $\left(\mathfrak{y}_{m}\right)_{m \in \mathbb{N}}$ respectively such that, for all $k \in \mathbb{N}, \mathfrak{x}_{n_{k}} \# \mathfrak{y}_{m_{k}}$.

(c) \# : $S^{\lambda} X \mapsto S^{\lambda} X$ is the lax colimit of $\left(\#: S^{\alpha} X \mapsto S^{\alpha} X\right)_{\alpha<\lambda}$.

The following result can be easily proven by transfinite induction.

Lemma. For all ordinals $\alpha$ and $\mathfrak{x}, \mathfrak{y} \in S^{\alpha} X$, we have $\mathfrak{x} \# \mathfrak{n}$ if and only if there exists some $\mathfrak{z} \in S^{\alpha} X$ such that $\mathfrak{z} \preccurlyeq \mathfrak{x}$ and $\mathfrak{z} \preccurlyeq \mathfrak{y}$.

Finally, we extend the diagonal relation $m_{X}$ to "higher order sequences". Recall that (4) is equivalent to (4a) and (4b), and (4b) is implied by (3'). Therefore it will be sufficient to consider $S e_{X}^{\circ}$ instead of $m_{X}$. For ordinals $\alpha$ and $\beta$ we define relations $m_{X}^{\alpha, \beta}: S^{\alpha} \longrightarrow S^{\beta}$ by putting

(a) $m_{X}^{\alpha, \beta}=0$ if $\alpha<\beta$,

(b) $m_{X}^{0,0}=\operatorname{id}_{X}$ and $m_{X}^{1,0}=\left(\preccurlyeq \cdot e_{X}\right)^{\circ}$,

(c) $m_{X}^{\alpha+1, \beta+1}=S m_{X}^{\alpha, \beta}$

(d) $m_{X}^{\lambda, \beta}=\left[m_{X}^{\alpha, \beta}\right]_{\alpha<\lambda}$,

(e) $m_{X}^{\lambda, \lambda}=\left\langle m_{X}^{\lambda, \beta}\right\rangle_{\beta<\lambda}$.

Hence $\left(x_{n}\right)_{n \in \mathbb{N}}\left(m_{X}^{1,0}\right) x$ if $\left\{n \in \mathbb{N} \mid x_{n}=x\right\}$ is infinite. In the sequel we will simply write $\mathfrak{x} \rightsquigarrow \mathfrak{y}$ instead of $\mathfrak{x}\left(m_{X}^{\alpha, \beta}\right) \mathfrak{y}$. It follows easily from (2) and (4a) 
that $\rightsquigarrow$ preserves convergence, that is, we have $\mathfrak{y} \rightarrow x$ whenever $\mathfrak{x} \rightarrow x$ and $\mathfrak{x} \rightsquigarrow \mathfrak{x}$.

Proposition. Let $Y$ be a set and $\mathfrak{y} \in S Y$ such that, for each $\mathfrak{x} \in S Y$, we have $\mathfrak{x} \# \mathfrak{y}$ or $\mathfrak{x} \rightsquigarrow x$ for some $x \in Y$. Then the following statements hold.

(a) For each $n \in \mathbb{N}(n \geq 1)$ and $\mathfrak{x} \in S^{n} Y$, we have $\mathfrak{x} \rightsquigarrow \mathfrak{x}^{\prime} \in S^{k} Y$ where

$$
k=0 \quad \text { or } \quad 1 \leq k \leq n \text { and } \mathfrak{x}^{\prime} \# e_{Y}^{1, k}(\mathfrak{y}) .
$$

(b) For each infinite ordinal $\alpha=\lambda+n$, with $n \in \mathbb{N}$ and $\lambda$ a limit ordinal, and each $\mathfrak{x} \in S^{\alpha} Y$, there exists some $\mathfrak{z}$ with $\mathfrak{x} \rightsquigarrow \mathfrak{z}$ where

$$
\mathfrak{z} \in S^{\alpha} Y \text { and } \mathfrak{z} \# e_{Y}^{1, \alpha}(\mathfrak{y}) \quad \text { or } \quad \mathfrak{z} \in S^{n} Y .
$$

Proof: To show (a), we prove by induction that, for each $n \geq 1$ and $\mathfrak{x} \in S^{n} Y$, we have $\mathfrak{x} \# e_{Y}^{1, n}(\mathfrak{y})$ or $\mathfrak{x} \rightsquigarrow \mathfrak{x}^{\prime} \in S^{n-1} Y$. For $n=1$ this is just the hypothesis. Assume now that the assertion is true for $n \geq 1$ and let $\left(\mathfrak{x}_{m}\right)_{m \in \mathbb{N}} \in S^{n+1} Y$. If $\mathfrak{x}_{m} \# e_{Y}^{1, n}(\mathfrak{y})$ for infinitely many $m \in \mathbb{N}$, then $\left(\mathfrak{x}_{m}\right)_{m \in \mathbb{N}} \# e_{Y}^{1, n+1}(\mathfrak{y})$. Otherwise we have $\mathfrak{x}_{m} \rightsquigarrow \mathfrak{x}_{m}^{\prime} \in S^{n-1} Y$ for almost all $m \in \mathbb{N}$. Hence $\left(\mathfrak{x}_{m}\right)_{m \in \mathbb{N}} \rightsquigarrow\left(\mathfrak{x}_{m}^{\prime}\right)_{m \in \mathbb{N}} \in$ $S^{n} Y$

To see (b), let $\mathfrak{x} \in S^{\alpha} Y$ and assume that the assertion is true for all infinite ordinals smaller then $\alpha$. Assume first that $\alpha=\lambda$ is a limit ordinal. Hence $\mathfrak{x} \in S^{\beta} Y$ for some $\beta<\lambda$ and the result follows from (a) (in case $\beta \in \mathbb{N}$ ) or from the induction hypothesis. Assume now that $\alpha=\lambda+n+1$ and $\mathfrak{x}=\left(\mathfrak{x}_{m}\right)_{m \in \mathbb{N}}$ with $\mathfrak{x}_{m} \in S^{\lambda+n} Y$ for each $m \in \mathbb{N}$.

1st case: $\quad$ For infinitely many $m \in \mathbb{N}, \mathfrak{x}_{m} \rightsquigarrow \mathfrak{z}_{m}$ where $\mathfrak{z}_{m} \# e_{Y}^{1, \lambda+n}(\mathfrak{y})$. Putting $\mathfrak{z}_{m}=\mathfrak{x}_{m}$ for all other $m \in \mathbb{N}$, we have $\left(\mathfrak{x}_{m}\right)_{m \in \mathbb{N}} \rightsquigarrow\left(\mathfrak{z}_{m}\right)_{m \in \mathbb{N}}$ and $(\mathfrak{z} m)_{m \in \mathbb{N}} \# e_{Y}^{1, \lambda+n+1}(\mathfrak{y})$.

2nd case: $\quad$ For almost all $m \in \mathbb{N}$ we have $\mathfrak{x}_{m} \rightsquigarrow \mathfrak{z}_{m} \in S^{n} Y$. Then $\left(\mathfrak{x}_{m}\right)_{m \in \mathbb{N}} \rightsquigarrow\left(\mathfrak{z}_{m}\right)_{m \in \mathbb{N}} \in S^{n+1} Y$

3.3. Theorem. Let $a: S X \rightarrow X$. Then $a \in \operatorname{Fix}_{\mathcal{C T}}$ if and only if a satisfies (1), (2), (3') and (4a).

Proof: Clearly, each $a \in \operatorname{Fix}_{\mathcal{C}}$ satisfies these axioms. To prove the converse, assume that $a: S X \longrightarrow X$ satisfies (1), (2), (3') and (4a). We have to show that $a$ satisfies $(*)$. Let $\left(x_{n}\right)_{n \in \mathbb{N}} \in S X, x \in X$ and assume that $\left(x_{n}\right)_{n \in \mathbb{N}}$ does not converges to $x$. Hence $\dot{x}_{n} \nrightarrow x$ for almost all $n \in \mathbb{N}$, and then without loss of generality we assume that it is true for all $n \in \mathbb{N}$. If $\left\{n \in \mathbb{N} \mid x_{n}=y\right\}$ is infinite for some $y \in X$, we chose $A=\{y\}$ and $\mathfrak{y}=\dot{y}$. Clearly we have 
$\forall \alpha \forall \mathfrak{z} \in S^{\alpha} A \mathfrak{z} \nrightarrow x$.

Assume now that $\left\{n \in \mathbb{N} \mid x_{n}=y\right\}$ is finite for each $y \in X$. We put $A=\left\{x_{n} \mid n \in \mathbb{N}\right\}$ and $\mathfrak{y}=\left(x_{n}\right)_{n \in \mathbb{N}}$. Note that $Y=A$ and $\mathfrak{y} \in S Y$ satisfy the hypothesis of Proposition 3.2. Let $\alpha$ be an ordinal and $\mathfrak{z} \in S^{\alpha} A$. Suppose that $\mathfrak{z} \rightarrow x$. From Proposition 3.2 we deduce that $\mathfrak{z} \rightsquigarrow y \in A$ or $\mathfrak{z} \rightsquigarrow \mathfrak{z}^{\prime} \# e_{A}^{1, \beta}(\mathfrak{y})$. The first case is impossible since $\dot{y} \nrightarrow x$. In the latter case there exists some $\mathfrak{z}^{\prime \prime}$ such that $\mathfrak{z}^{\prime \prime} \preccurlyeq \mathfrak{z}^{\prime}$ and $\mathfrak{z}^{\prime \prime} \preccurlyeq e_{A}^{1, \beta}(\mathfrak{y})$. But then $\mathfrak{z}^{\prime \prime} \rightarrow x$ and $\mathfrak{z}^{\prime \prime} \rightsquigarrow \mathfrak{y}=\left(x_{n}\right)_{n \in \mathbb{N}}$, hence $\left(x_{n}\right)_{n \in \mathbb{N}} \rightarrow x$ which contradicts our assumption $\left(x_{n}\right)_{n \in \mathbb{N}} \nrightarrow x$.

\section{Further notes}

4.1. A new diagonal. Axiom (4) is a very natural diagonal axiom in this context, but Theorem 3.3 shows that we do not need all of its strength. Instead of (4), one uses (4a). To keep, simultaneously, condition (4a) and the parallelism with the ultrafilter case, one might use just a particular case as definition of diagonal sequence. That is, $\mathfrak{x}=\left(x_{n}\right)_{n \in \mathbb{N}} \in S X$ is a diagonal sequence of $\mathfrak{X}=\left(x_{m}^{n}\right)_{n, m \in \mathbb{N}} \in S S X$ (we write $\mathfrak{X}\left(n_{X}\right) \mathfrak{x}$ ) if there is a $k \in \mathbb{N}$ such that, for all $n \geq k,\left[\dot{x}_{n}\right]=\left[\left(x_{m}^{n}\right)_{m \in \mathbb{N}}\right]$. Defining the co-Kleisli composition * with respect to the new diagonal $n_{X}$, we can rewrite condition (4a) as $a * a \leq a$.

Notice that $\mathfrak{X} \in S S X$ has a diagonal, in the new sense, if and only if $\mathfrak{X}=\left[\left(\dot{x}_{n}\right)_{n \in \mathbb{N}}\right]$ for a sequence $\left(x_{n}\right)_{n \in \mathbb{N}}$. With this approach we recover the uniqueness of the diagonal, if exists. This happens mainly because most of the elements of $S S X$ have no diagonal sequence.

4.2. Fréchet spaces. We have seen in Section 2.5 that our axioms fulfil well the propose of describing Fréchet spaces. Axiom (5) introduced there is closer to our intuitive idea of diagonal sequence than Axiom (4) and in its presence (4a) can be replaced by a weaker condition.

Proposition. Under (1), (2), (3) and (5), axiom (4a) is equivalent to $\left(4^{-}\right)\left(\dot{x}_{n}\right)_{n \in \mathbb{N}} \rightarrow \dot{x} \Rightarrow\left(x_{n}\right)_{n \in \mathbb{N}} \rightarrow x$.

Condition $\left(4^{-}\right)$might also be written in terms of co-Kleisli composition with respect to $n_{X}$, i.e. it is equivalent to $e_{X}^{\circ} * a \leq a$.

Proof: Clearly (4a) implies $\left(4^{-}\right)$. Let $\left(x_{n}\right)_{n \in \mathbb{N}}$ be a sequence such that there is $\left(y_{n}\right)_{n \in \mathbb{N}}$ with $\left(\dot{x}_{n}\right)_{n \in \mathbb{N}} \rightarrow\left(y_{n}\right)_{n \in \mathbb{N}} \rightarrow x$. We want to show that $\left(x_{n}\right)_{n \in \mathbb{N}}$ 
converges to $x$. According to (2) and (3), it is only necessary to prove that a subsequence of $\left(x_{n}\right)_{n \in \mathbb{N}}$ converges to $x$. Axiom (5) implies that either $\left(x_{n}\right)_{n \in \mathbb{N}}$ has a convergent subsequence to $x$ or an infinite number of the members of the sequence must converge to $x$. In the second case, $\left(4^{-}\right)$implies the desired convergence.

Corollary. A convergence space is a convergence of a topological Fréchet $T_{1}$ space if and only if satisfies (1), (2), (3), (5) and the constant sequences have unique limits (that is $\operatorname{id}_{X}=a \cdot e_{X}$ ).

4.3. Pretopological spaces. There are several equivalent ways of describing pretopological spaces. Here we use the one with closure operators. We say that $(X, k)$ is a pretopological space if $k$ is grounded $(k(\emptyset)=\emptyset)$, extensive $(A \subseteq k(A))$, and additive $(k(A \cup B)=k(A) \cup k(B))$. A sequence $\left(x_{n}\right)_{n \in \mathbb{N}}$ converges to $x$ if, for every $\left(y_{n}\right)_{n \in \mathbb{N}} \preccurlyeq\left(x_{n}\right)_{n \in \mathbb{N}}, x \in k\left(\left\{y_{n} \mid n \in \mathbb{N}\right\}\right)$. Now we will see which convergence spaces are induced by pretopologies. Clearly, among them are the ones coming from a topology. As for topological spaces, they are the fixed points of a Galois correspondence, namely of

$$
\left\{k \in P X^{P X} \mid k \text { is extensive }\right\} \underset{\mathcal{C}}{\longleftarrow}\{a: S X \rightarrow X \mid a \text { satisfies (1),(2) }\} .
$$

Theorem. ([Kou85]) Let $a: S X \longrightarrow X$ be a convergence in $X$ satisfying (1) and (2). Then $a \in \operatorname{Fix}_{\mathcal{C} \sigma}$ if and only if it satisfies (3) and (4-).

This corresponds exactly to the ultrafilter case, where the fixed points of the corresponding Galois correspondence are characterized as those $a$ : $U X \mapsto X$ which satisfy $e_{X}^{\circ} * a \leq a$ (see [Hof05a]).

Corollary. ([Čec66]) A convergence space is a convergence of a pretopological $T_{1}$-space if and only if satisfies (1), (2), (3) and the constant sequences have unique limits.

Remark. Since for sequential spaces the sequential closure need not be idempotent, they are not fixed points of this Galois correspondence via the natural embedding from Top to PrTop. However, the sequential closure provides an embedding from $\operatorname{Fix}_{\sigma \mathcal{T}}$ to $\mathrm{Fix}_{\sigma \mathcal{C}}$. The topological spaces characterized by sequential convergence in both ways are exactly the sequential spaces with idempotent sequential closure, that is the Fréchet spaces. 


\section{References}

[Bar70] Michael Barr, Relational algebras, Reports of the Midwest Category Seminar, IV, Lecture Notes in Mathematics, Vol. 137. Springer, Berlin, 1970, pp. 39-55.

[Bro74] Ronald Brown, On sequentially proper maps and a sequential compactification, J. London Math. Soc. (2) 7 (1974), 515-522.

[Car37a] Henri Cartan, Filtres et ultrafiltres, C. R. Acad. Sci. Paris 205 (1937), 777-779.

[Car37b] — Théorie des filtres, C. R. Acad. Sci. Paris 205 (1937), 595-598.

[Čec66] Eduard Čech, Topological spaces, Revised edition by Zdeněk Frolík and Miroslav Katětov. Scientific editor, Vlastimil Pták. Editor of the English translation, Charles O. Junge, Publishing House of the Czechoslovak Academy of Sciences, Prague, 1966.

[CH04] Maria M. Clementino and Dirk Hofmann, Effective descent morphisms in categories of lax algebras, Appl. Categ. Structures 12 (2004), no. 5-6, 413-425.

[CH05] , Exponentiation in $\boldsymbol{V}$-categories, Topology Appl., to appear (2005).

[CHT04] Maria M. Clementino, Dirk Hofmann and Walter Tholen, One setting for all: metric, topology, uniformity, approach structure, Appl. Categ. Structures 12 (2004), no. 2, $127-$ 154.

[CT03] Maria M. Clementino and Walter Tholen, Metric, topology and multicategory - a common approach, J. Pure Appl. Algebra 179 (2003), no. 1-2, 13-47.

[Dud64] R. M. Dudley, On sequential convergence, Trans. Amer. Math. Soc. 112 (1964), 483-507.

[Eng89] Ryszard Engelking, General topology, second ed., Sigma Series in Pure Mathematics, vol. 6, Heldermann Verlag, Berlin, 1989, Translated from the Polish by the author.

[Hof05a] Dirk Hofmann, An algebraic description of regular epimorphisms in topology, J. Pure Appl. Algebra 199 (2005), no. 1-3, 71-86.

[Hof05b] _ Exponentiation for unitary structures, Topology Appl., to appear (2005).

[HT04] Dirk Hofmann and Walter Tholen, Kleisli compositions for topological spaces, preprint.

[Kis60] J. Kisyński, Convergence du type $\mathcal{L}$, Colloq. Math. 7 (1959/1960), 205-211.

[Kou85] Václav Koutník, Closure and topological sequential convergence, Convergence structures 1984 (Bechyně, 1984), Math. Res., vol. 24, Akademie-Verlag, Berlin, 1985, pp. 199-204.

[Kow54] Hans-Joachim Kowalsky, Limesräume und Komplettierung, Math. Nachr. 12 (1954), 301340.

[Man02] Ernest G. Manes, Taut monads and T0-spaces, Theoret. Comput. Sci. 275 (2002), no. 1-2, 79-109.

[Sea05] Gavin J. Seal, Canonical and op-canonical lax algebras, Theory Appl. Categ. 14 (2005), 221-243 (electronic).

[SM22] H. L. Smith and E. H. Moore, A General Theory of Limits, Amer. J. Math. 44 (1922), no. $2,102-121$.

[Wy196] Oswald Wyler, Convergence axioms for topology, Papers on general topology and applications (Gorham, ME, 1995), Ann. New York Acad. Sci., vol. 806, New York Acad. Sci., New York, 1996, pp. 465-475.

Gonçalo Gutierres

Departamento de Matemática, Universidade de Coimbra, 3001-454 Coimbra, Portugal

E-mail address: ggutc@mat.uc.pt

Dirk HOFMANN

Departamento de Matemática, Universidade de Aveiro, 3810-193 Aveiro,Portugal

E-mail address: dirk@mat.ua.pt 